International Journal of Pure and Applied Mathematics

Volume 93 No. 4 2014, 501-509

ISSN: 1311-8080 (printed version); ISSN: 1314-3395 (on-line version)

url: http://www.ijpam.eu

doi: http://dx.doi.org/10.12732/ijpam.v93i4.2

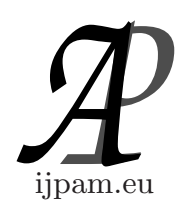

\title{
HARMONIOUS AND VERTEX GRACEFUL LABELING ON PATH AND STAR RELATED GRAPHS
}

\author{
P. Selvaraju ${ }^{1}$, P. Balaganesan ${ }^{2}$, J. Renuka ${ }^{3}$, M.L. Suresh ${ }^{4}$ \\ ${ }^{1}$ Department of Mathematics \\ Vel Tech. Multi Tech. Dr. Rangarajan Dr. Sankanthula \\ Engineering College \\ Avadi, Chennai, 600 062, INDIA \\ ${ }^{2,4}$ Department of Mathematics \\ Hindustan University \\ Chennai, 603 103, INDIA \\ ${ }^{3}$ Departments of Mathematics \\ Sri Sai Ram Engineering College \\ INDIA
}

\begin{abstract}
In this paper, we show that $B^{2}(n, n)$ is harmonious [4],$P_{i}^{n}$ is harmonious [3], $P_{n} \times C_{m}$ is vertex graceful for $n \geq 2$, and $m \geq 5, m$ is odd, $B^{2}(n, n)$ is vertex graceful [4], $P_{i}^{n}$ is vertex graceful, [3], $L_{n} \circ K_{1}$ is vertex graceful $\forall n$ and $P_{n} \times P_{2}$ is vertex graceful, $n$ is odd [3].
\end{abstract}

AMS Subject Classification: $05 \mathrm{C} 78$

Key Words: harmonious graphs, harmonious labeling, vertex graceful graphs, vertex graceful labeling, bi-stars, path, Cartesian product

\section{Introduction}

Graph labeling, where the vertices are assigned values subject to certain conditions have often been motivated by practical problems. Labelled graphs serves as useful mathematical models for a broad range of applications such as coding

Received: October 23, 2013

(C) 2014 Academic Publications, Ltd.

$\S_{\text {Correspondence author }}$ url: www.acadpubl.eu 
theory, including the design of good radar type codes, synch-set codes, missile guidance codes and convolution codes with optimal autocorrelation properties. They facilitate the optimal non standard encoding of integers.

All graphs in this paper are finite, simple graphs with no loops or multiple edges. The symbols $V(G)$ and $E(G)$ denote the vertex set and edge set of the graph $\mathrm{G}$. A graph with p vertices and q edges is called $\mathrm{G}(\mathrm{p}, \mathrm{q})$ graph. Harmonious graphs naturally arose in the study by Graham and Sloane [1] of modular version of additive base problems stemming from error correction codes. They obtained some graphs are harmonious.

Definition 1.1. A Graph $G$ is said to be harmonious if there exist an injection $f: V(G) \rightarrow Z_{q}$ such that the induced function $f^{*}: E(G) \rightarrow Z_{q}$ defined by $f^{*}(u v)=(f(u)+f(v))(\bmod q)$ is a bijection from $E(G)$ onto $Z_{q}$ then, $f$ is said to be harmonious labeling of $G$.

Definition 1.2. A graph $G$ with $p$ vertices and $q$ edges is said to be vertex graceful if a labeling $f: V(G) \rightarrow\{1,2,3 \ldots p\}$ exists in such a way that the induced labeling $f^{*}: E(G) \rightarrow Z_{q}$ defined by $f^{*}((u, v))=f(u)+f(v)(\bmod q)$ is a bijection from $E(G)$ onto $Z_{q}$. The concept of vertex graceful was introduced by Lee, Pan and Tsai in 2005.

Definition 1.3. For a simple connected graph $G$ the Square of graph $G$ is denoted by $G^{2}$ and defined as the graph with the same vertex set as of $G$ and two vertices are adjacent in $G^{2}$ if they are at a distance 1 or 2 apart in $G$.

\section{Main Results of Harmonious Labeling on Path and Star Related Graphs}

Theorem 2.1. The graph $B^{2}(n, n)$ is harmonious $\forall n$.

Proof. Consider $B^{2}(n, n)$ with the vertex set $\left\{u, v, u_{i}, v_{i}, 1 \leq i \leq n\right\}$ where $u_{i}, v_{i}$ are the pendant vertices. Let $G$ be the graph $B^{2}(n, n)$, then $|V(G)|=2 n+2$ and $|E(G)|=4 n+1$. We define the vertex labeling $f: V(G) \rightarrow\{0,1,2,3 \ldots,(q-1)\}$ as follows:

$$
\begin{aligned}
& v=0, u=2 n+1 \\
& v_{i}=i, 1 \leq i \leq n \\
& u_{i}=n+i, 1 \leq i \leq n
\end{aligned}
$$

Let $A, B, C, D$ denote edge set.

$$
A=\left\{e_{i}=v v_{i} / e_{i}=i: 1 \leq i \leq n\right\}
$$




$$
\begin{aligned}
& B=\left\{e_{i}=u v_{i} / e_{i}=(2 n+i+1)(\bmod q): 1 \leq i \leq n\right\} \\
& C=\left\{e_{i}=v u_{i} / e_{i}=(n+i)(\bmod q): 1 \leq i \leq n\right\} \\
& D=\left\{e_{i}=u u_{i} / e_{i}=(3 n+i+1)(\bmod q): 1 \leq i \leq n\right\}
\end{aligned}
$$

It is clear that vertex set labeling and edge set labeling are distinct. Hence, the $B^{2}(n, n)$ is harmonious graph $\forall n$.

Illustration 2.2. A harmonious graph $B^{2}(7,7)$ is shown in the figure 1 .

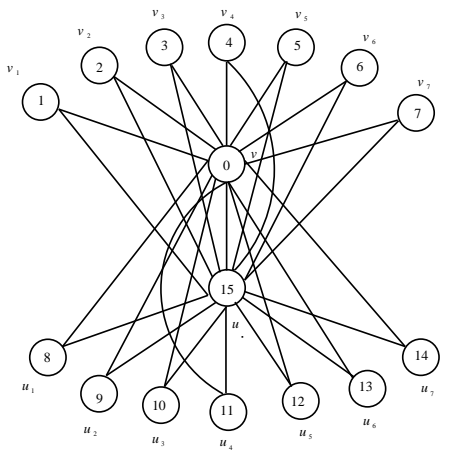

Figure 1: The graph $B^{2}(7,7)$

Remark 2.3. Let $\alpha$ be the collection of paths $P_{i}^{n}$, where $n$ is odd, and $P_{i}^{n}=u_{1}^{i}, u_{2}^{i}, \ldots, u_{n}^{i}, 1 \leq i \leq m$. Let $G$ be the graph obtained from $\alpha$ with $V(G)=\cup_{i=1}^{m} V\left(p_{n}^{i}\right)$ and $E(G)=\cup_{i=1}^{m} E\left(p_{n}^{i}\right) \cup\left(u_{\frac{\mathrm{n}+1}{2}}^{i} u_{\frac{\mathrm{n}+1}{2}}^{i+1}\right)$

Theorem 2.4. The graph $P_{i}^{n}$ is harmonious graph, $\forall n$.

Proof. Let $G=P_{i}^{n}$ be a graph with $p=2 n$ vertices and $q=(2 n-1)$. The required vertex labeling $f: V(G) \rightarrow\{0,1,2, \cdots, q-1\}$ is as follows:

case $(\mathbf{i}): \mathrm{n}$ is odd

$$
u_{j}^{i}=n(i-1)+j-1 ; 1 \leq j \leq n, i=1,2 .
$$

Let $A$ and $B$ denote edge set.

$$
\begin{aligned}
& A=\left\{e_{j}^{1}=u_{j}^{1} u_{j+1}^{2} / e_{i}^{1}=(2 n(i-1)+2 j-1)(\bmod q): 1 \leq j \leq n-1, i=1,2\right\} \\
& B=\left\{e_{\frac{\mathrm{n}+1}{2}}^{1}=u_{\frac{\mathrm{n}+1}{2}}^{1} u_{\frac{\mathrm{n}+1}{2}}^{2} / e_{\frac{\mathrm{n}+1}{2}}^{1}=(2 n+1)(\bmod q)\right\}
\end{aligned}
$$

case(ii): $n$ is even

$$
\begin{aligned}
& u_{j}^{i}=n(i-1)+j-1 ; 1 \leq j \leq n, 1 \leq i \leq m, i \text { is odd } \\
& u_{j}^{i}=n i-j ; 1 \leq j \leq n, 1 \leq i \leq m, i \text { is even }
\end{aligned}
$$

Let $A, B, C$ are denote edge set. 
$A=\left\{e_{i}^{j}=u_{j}^{i} u_{j}^{i+1} / e_{j}^{i}=(2 n(i-1)+2 j-1)(\bmod q): 1 \leq j \leq n-1,1 \leq i \leq m, \mathrm{i}\right.$ is odd $\}$

$B=\left\{e_{j}^{i}=u_{j}^{i} u_{j}^{i+1} / e_{j}^{i}=(2(n i-j)-1)(\bmod q): 1 \leq j \leq n-1,1 \leq i \leq m, \quad i\right.$ is even $\}$

$C=\left\{e_{\frac{\mathrm{n}}{2}}^{i}=u_{\frac{\mathrm{n}}{2}}^{i} u_{\frac{\mathrm{n}}{2}}^{i+1} / e_{\frac{\mathrm{n}}{2}}^{i}:=(2 n i-1)(\bmod q)\right\}$

It is clear that vertex set labeling and edge set labeling are distinct. Hence, the graph $G$ is harmonious graph $\forall n$.

Illustration 2.5. A harmonious graph $P_{i}^{5}(i=1,2)$ is shown in the figure 2 .

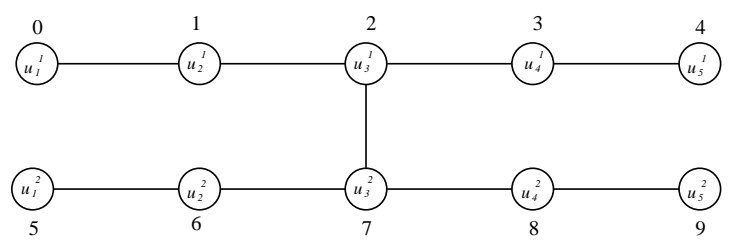

Figure 2: The graph $P_{i}^{5}(i=1,2)$

\section{Main Results of Vertex Graceful labeling on Path and Star Related Graphs}

Theorem 3.1. The graph $P_{n} \times C_{m}$ is a vertex graceful graph, $\forall n, n \geq 2$ and $m \geq 5, m$ is odd.

Proof. Consider the graph $G=P_{n} \times C_{m}$ with $n m$ vertices and $q=(2 n-1) m$ edges. Suppose that the vertices $v_{i}^{j} ; 1 \leq i \leq m$ and $j=0,1,2, \cdots, n$ of the cycle $C_{m}$ run consecutively with $v_{1}^{j}$ joined to $v_{m}^{j}$. The required vertex labeling $f: V(G) \rightarrow 1,2, \cdots, p$ is as follows:

$$
v_{i}^{j}= \begin{cases}\frac{n-1}{2}+\frac{1+i}{2}+n j ; & 1 \leq i \leq n \text { and } i \text { is odd, } 1 \leq j \leq m \\ \frac{1+i}{2}+\frac{n j}{2} ; & \text { and } j \text { is odd } \\ & 1 \leq i \leq n \text { and } i \text { is odd and } \\ & j=0,2,4, \cdots, n\end{cases}
$$




$$
v_{i}^{j}= \begin{cases}\frac{n+1+i}{2}-\frac{n+1}{2}+n j ; & 1 \leq i \leq n \text { and } i \text { is odd, } 1 \leq j \leq n \\ & \text { and } j \text { is odd } \\ \frac{n+1+i}{2}+\frac{n j}{2} ; & 1 \leq j \leq n \text { and } i \text { is odd and } \\ & j=0,2,4, \cdots, n\end{cases}
$$

Let $\mathrm{A}, \mathrm{B}, \mathrm{C}$ are denote the edge set.

$A=\left\{e_{i}^{j}=v_{i}^{j} v_{i}^{j+1}=\left(\frac{3 n+1}{2}+2 n j+i\right)(\bmod q) / 1 \leq i \leq n, j=0,1,2, \cdots, n-\right.$ $1\}$

$B=\left\{e_{i}^{j}=v_{i}^{j} v_{k}^{j}=\left(\frac{n+1}{2}+2 n j+k\right)(\bmod q) / k=(1+i)(\bmod n), 1 \leq i \leq\right.$ $n, j=0,2,4,, n\}$

$C=\left\{e_{i}^{j}=v_{i}^{j} v_{i+1}^{j}=\left(\frac{n+1}{2}+2 n j+i\right)(\bmod q) / 1 \leq i \leq n, 1 \leq j \leq m\right.$ and $j$ is odd $\}$ It is clear that vertex set labeling and edge set labeling are distinct. Hence, the $P_{n} \times C_{m}$ is a vertex graceful graph, for $n \geq 2$ and $m \geq 5, m$ is odd.

Illustration 3.2. A vertex graceful graph $P_{3} \times C_{5}$ is shown in the figure 3.

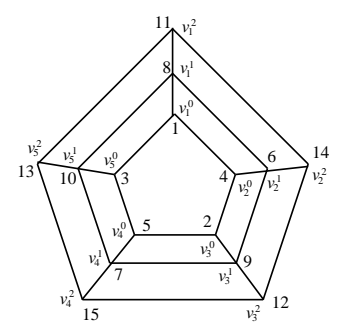

Figure 3: The graph $P_{3} \times C_{5}$

Theorem 3.3. The graph $B^{2}(n, n)$ is vertex graceful graph $\forall n$.

Proof. Consider $B^{2}(n, n)$ with the vertex set $\left\{u, v, u_{i}, v_{i}, 1 \leq i \leq n\right\}$ where $u_{i}, v_{i}$ are the pendant vertices. Let $G$ be the graph $B^{2}(n, n)$ then $|V(G)|=2 n+2$ and $|E(G)|=4 n+1$. We define the vertex labeling $f: V(G) \rightarrow\{1,2, \ldots, p\}$ as follows.

case $(\mathbf{i}): \mathrm{n}$ is odd

$$
\begin{aligned}
& v=1, u=2 n+2 \\
& v_{i}=i+1,1 \leq i \leq n
\end{aligned}
$$


Let $A, B, C, D$ denote edge set.

$$
u_{i}=n+i+1,1 \leq i \leq n .
$$

$A=\left\{e_{i}=v v_{i} / e_{i}=i+2: 1 \leq i \leq n\right\}$

$B=\left\{e_{i}=u v_{i} / e_{i}=(2 n+i+3)(\bmod q): 1 \leq i \leq n\right\}$

$C=\left\{e_{i}=v u_{i} / e_{i}=(n+i+2)(\bmod q): 1 \leq i \leq n\right\}$

$D=\left\{e_{i}=u u_{i} / e_{i}=(3 n+i+3)(\bmod q): 1 \leq i \leq n\right\}$

case(ii): $\mathrm{n}$ is even

$u_{j}^{i}=n(i-1)+j ; 1 \leq j \leq n, 1 \leq i \leq m$, i is odd

$u_{j}^{i}=n i-j+1 ; 1 \leq j \leq n, 1 \leq i \leq m$, i is even

Let $A, B, C$ are denote edge set.

$A=\left\{e_{i}^{j}=u_{j}^{i} u_{j}^{i+1} / e_{j}^{i}=(2 n(i-1)+2 j+1)(\bmod q): 1 \leq j \leq n-1,1 \leq i \leq m, i\right.$ is odd $\}$

$B=\left\{e_{j}^{i}=u_{j}^{i} u_{j}^{i+1} / e_{j}^{i}=(2(n i-j)+1)(\bmod q): 1 \leq j \leq n-1,1 \leq i \leq m, \quad i\right.$ is even $\}$

$C=\left\{e_{\frac{\mathrm{n}}{2}}^{i}=u_{\frac{\mathrm{n}}{2}}^{i} u_{\frac{\mathrm{n}}{2}}^{i+1} / e_{\frac{\mathrm{n}}{2}}^{i}:=(2 n i+1)(\bmod q)\right\}$

It is clear that vertex set labeling and edge set labeling are distinct. Hence, the $B^{2}(n, n)$ is vertex graceful graph.

Illustration 3.4. A vertex graceful graph $B^{2}(7,7)$ is shown in the figure 4.

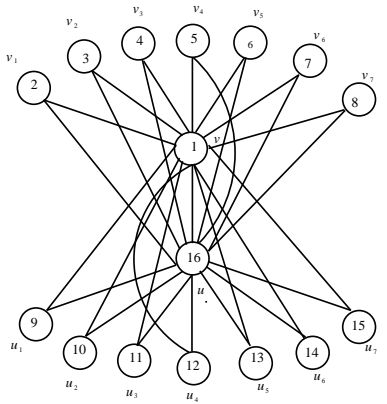

Figure $4: B^{2}(7,7)$

Theorem 3.5. The graph $P_{i}^{n}$ is vertex graceful graph $\forall n, n$ is odd.

Proof. Let $G=P_{n}^{i}$ be a graph with $p=2 n$ vertices and $q=(2 n-1)$. The required vertex labeling $f: V(G) \rightarrow\{1,2, \ldots, p\}$ is as follows:

$\operatorname{case}(\mathbf{i}): \mathrm{n}$ is odd 
Let $A$ and $B$ denote edge set.

$$
u_{j}^{i}=n(i-1)+j ; 1 \leq j \leq n, i=1,2 .
$$

$A=\left\{e_{j}^{i}=u_{j}^{i} u_{j+1}^{i} / e_{j}^{i}=(2 n(i-1)+2 j+1)(\bmod q): 1 \leq j \leq n-1\right.$, $i=1,2\}$

$B=\left\{e_{\frac{n+1}{2}}^{1}=u_{\frac{n+1}{2}}^{1} u_{\frac{n+1}{2}}^{2} / e_{\frac{n+1}{2}}^{1}=(2 n+1)(\bmod q)\right\}$

It is clear that vertex set labeling and edge set labeling are distinct. Hence the graph $G$ vertex graceful forall $n, n$ is odd.

case(ii): $n$ is even, $1 \leq i \leq m$

$$
\begin{aligned}
& u_{j}^{i}=n(i-1)+j ; 1 \leq j \leq n, 1 \leq i \leq m, \mathrm{i} \text { is odd } \\
& u_{j}^{i}=n i-j+1 ; 1 \leq j \leq n, 1 \leq i \leq m, \mathrm{i} \text { is even }
\end{aligned}
$$

Let $A, B, C$ are denote edge set.

$A=\left\{e_{i}^{j}=u_{j}^{i} u_{j}^{i+1} / e_{j}^{i}=(2 n(i-1)+2 j+1)(\bmod q): 1 \leq j \leq n-1,1 \leq i \leq m, i\right.$ is odd $\}$

$B=\left\{e_{j}^{i}=u_{j}^{i} u_{j}^{i+1} / e_{j}^{i}=(2(n i-j)+1)(\bmod q): 1 \leq j \leq n-1,1 \leq i \leq m\right.$, $i$ is even $\}$

$C=\left\{e_{\frac{\mathrm{n}}{2}}^{i}=u_{\frac{\mathrm{n}}{2}}^{i} u_{\frac{\mathrm{n}}{2}}^{i+1} / e_{\frac{\mathrm{n}}{2}}^{i}:=(2 n i+1)(\bmod q)\right\}$

It is clear that vertex set labeling and edge set labeling are distinct. Hence, the graph $G$ is vertex graceful graph $\forall n$.

Illustration 3.6. A vertex graceful graph $P_{5}^{i}(i=1,2)$ is shown in the figure 5 .

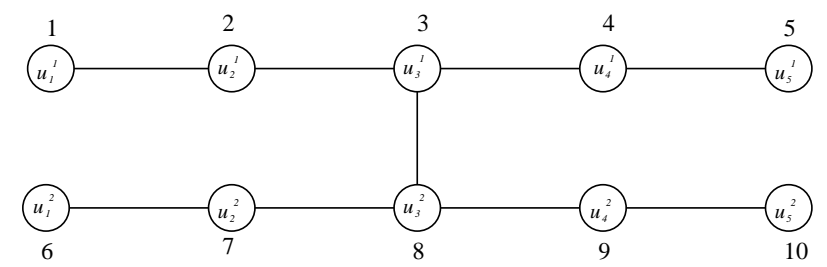

Figure 5: $P_{5}^{i}(i=1,2)$

Definition 3.7. The graph $L_{n}=P_{n} \times P_{2}$ is called the ladder.

Theorem 3.8. The graph $L_{n} \circ K_{1}$ is a vertex graceful $\forall n$.

Proof. consider the graph $G=L_{n} \circ K_{1}$. Let $V\left(L_{n}\right)=u_{i}, v_{i}: 1 \leq i \leq n$. $E\left(L_{n}\right)=u_{i} v_{i}=1 \leq i \leq n-1 \cup u_{i} u_{i+1}: 1 \leq i \leq n-1$ 
$\cup v_{i} v_{i+1}: 1 \leq i \leq n-1$. Let $w_{i}$ be pendent vertex adjacent to $\mathrm{u}_{i}$ and let $z_{i}$ be the pendent vertex adjacent to $v_{i}$. The required vertex labeling $f: V(G) \rightarrow$ $\{1,2, \ldots, p\}$ is as follows:

$$
\begin{aligned}
& u_{i}=5 i-4 ; 1 \leq i \leq n \\
& v_{i}=5 i-3 ; 1 \leq i \leq n \\
& w_{i}=5 i-5 ; 1 \leq i \leq n \\
& z_{i}=5 i-2 ; 1 \leq i \leq n
\end{aligned}
$$

Let $A, B, C, D, E$ denote the edge set.

$A=\left\{e_{i}=u_{i} w_{i} / e_{i}=10 i-9(\bmod q): 1 \leq i \leq n\right\}$,

$B=\left\{e_{i}=u_{i} v_{i} / e_{i}=10 i-7(\bmod q), 1 \leq i \leq n\right\}$

$C=\left\{e_{i}=v_{i} z_{i} / e_{i}=10 i-5(\bmod q): 1 \leq i \leq n\right\}$,

$D=\left\{e_{i}=u_{i} u_{i+1} / e_{i}=10 i-3(\bmod q): 1 \leq i \leq n\right\}$

$E=\left\{e_{i}=v_{i} v_{i+1} / e_{i}=10 i-1(\bmod q): 1 \leq i \leq n\right\}$

It is clear that vertex set labeling and edge set labeling are distinct. Then the graph $G=L_{n} \circ K_{1}$ is vertex graceful $\forall n$.

Theorem 3.9. The graph $P_{n} \times P_{2}$ is a vertex graceful $\forall n, n$ is odd

Proof. Consider the graph $G=P_{n} \times P_{2}$ with $2 n$ vertices and $q=3 n-2$ edges. Let $v_{1 j}$ and $v_{2 j}$ be the first and second row vertices of $G$ respectively for $1 \leq j \leq n$. The required vertex labeling $f: V(G) \rightarrow\{1,2, \cdots, p\}$ is as follows:

$$
\begin{aligned}
& v_{1 j}=\frac{j+1}{2}, 1 \leq j \leq n ; \mathrm{j} \text { is odd } \\
& v_{1 j}=\frac{n+j+1}{2}, 1 \leq j \leq n ; \mathrm{j} \text { is even } \\
& v_{2 j}=\frac{3 n+j}{2}, 1 \leq j \leq n ; \mathrm{j} \text { is odd } \\
& v_{2 j}=\frac{2 n+j}{2}, 1 \leq j \leq n ; \mathrm{j} \text { is even }
\end{aligned}
$$

Let $A, B, C$ denote edge set.

$$
\begin{aligned}
& A=\left\{e_{j}=v_{1 j} v_{1 j+1} / e_{j}=\frac{n+2 j+3}{2}(\bmod q): 1 \leq j \leq n-1\right\} \\
& B=\left\{e_{j}=v_{2 j} v_{2 j+1} / e_{j}=\frac{5 n+2 j+1}{2}(\bmod q): 1 \leq j \leq n-1\right\} \\
& C=\left\{e_{j}=v_{1 j} v_{2 j} / e_{j}=\frac{3 n+1+2 j}{2}(\bmod q): 1 \leq j \leq n\right\}
\end{aligned}
$$

It is clear that vertex set labeling and edge set labeling are distinct. Hence, the graph $P_{n} \times P_{2}$ is a vertex graceful $\forall n, n$ is odd.

Illustration 3.10. A vertex graceful graph $P_{7} \times P_{2}$ is shown in the figure 6. 


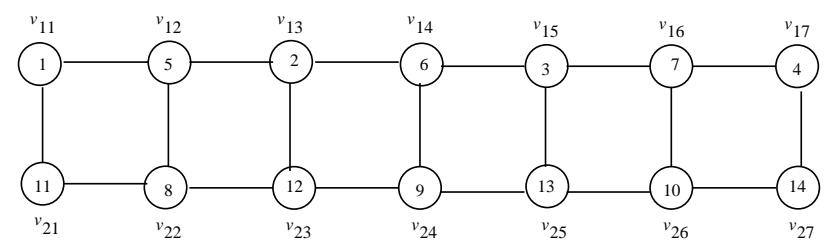

Figure 6: The graph $P_{7} \times P_{2}$

\section{References}

[1] J.A. Gallian, A Dynamic Survey of Graph Labeling, Electronic. J. Combinatorics.

[2] Harary, F. 1972 Graph Theory Addison Wesley, Mass Reading.

[3] Ponraj. R, Studies in labeling and graphs, Ph. D., Thesis of Manonmaniam Sundaranar University, India ,September 2004.

[4] S.K Vaidya , N H shah, Graceful and odd graceful labeling of some graphs,International journal of soft computing,Vol.3,No. 1(2013) 61-68.

[5] S.K Vaidya, N H shah, Some new odd harmonious graphs, international journal of Mathematics and soft computing, Vol. I, No.1 (2011) , 9-16. 
